\title{
Sequence and transcript analyses of antioxidant genes from Acanthus ebracteatus Vahl.
}

\begin{abstract}
In this study, we report the sequence, Southern analyses and spatial distribution of four cDNA sequences related to reactive oxygen species (ROS) scavenging systems from a mangrove plant, Acanthus ebracteatus. These four complementary DNA (cDNA) sequences encode cytosolic ascorbate peroxidase (AeAPX), monodehydroascorbate reductase (AeMDHR), glutathione-S-transferase (AeGST), and mitochondrial manganese superoxide dismutase (AeMnSOD), respectively. Experimental results indicated that AeAPX and AeGST belong to multigene families, whereas AeMDHAR and AeMnSOD exhibited substantial differences from other members of the same families. Transcript analyses indicated that all these genes were expressed in flowers. However, only AeAPX, AeMDHAR, and AeMnSOD were expressed in all tissues examined. Although both AeMDHAR and AeMnSOD were highly expressed in flowers, the highest expression of AeMnSOD was in the leaf tissue. The expression of AeMDHAR and AeMnSOD in stem was slightly higher than in the root. AeAPX was expressed at low levels in root and stem tissues and its expression in leaf was slightly higher than in the flower. On the other hand, the transcripts of AeGST were not detected in root and leaf. Its expression was almost equal in stem and flower. The information on spatial distribution and predicted subcellular locations of these antioxidant proteins are important for comprehensive analysis and characterization of ROS scavenging and signaling mechanisms in various plant compartments and tissues.
\end{abstract}

Keyword: Acanthus ebracteatus, Mangrove, Reactive oxygen species, Subcellular localization, Transcript analysis 